\title{
Sublobar resection versus ablation for stage I non-small-cell lung cancer: a meta-analysis
}

\author{
Yong Li ${ }^{1}$, Fang Yang ${ }^{1}$, Ya-Yong Huang ${ }^{2^{*}}$ and Tao Wang ${ }^{2}$
}

\begin{abstract}
Background: Stage I non-small-cell lung cancer (NSCLC) can be treated by both ablation and sublobar resection (SR). This meta-analysis was therefore designed to better compare the relative safety and efficacy of these two approaches to treating stage I NSCLC.

Materials and methods: Relevant studies published through November 2020 in the Cochrane Library, Embase, and PubMed databases were identified for analyses which were conducted with RevMan v5.3.

Results: In total, 816 potentially relevant articles were identified, of which 8 were ultimately included in the final meta-analysis. Patients in the SR group exhibited a signficantly lower pooled local recurrence (LR) rate (5.0\% vs. 25.4\%, $P<0.0001)$, although pooled distant recurrence (DR) rates were similar in both groups $(25.7 \% \mathrm{vs} .23 .1 \%, P=0.75)$. The pooled hazard ratio (HR) for overall survival (OS) (HR: 1.23; 95\% Cl: 1.13-1.33, $P<0.00001)$, progression-free survival (PFS) (HR: $1.34 ; 95 \% \mathrm{Cl}: 1.15-1.55, P=0.0002)$, and cancer-specific survival (HR: 1.39; 95\% Cl: 1.15-1.70, $P=0.0009$ ) all indicated better survival outcomes among patients that underwent $\mathrm{HR}$ treatment, while pooled complication rates were similar in both groups ( $27.7 \%$ vs. $43.8 \%, P=0.27)$. Patients that underwent ablation exhibited significantly shorter pooled post-operative hospitalization relative to those in the SR group (MD: 5.93; 95\% Cl: 0.78-11.07, $P=0.02$ ). No evidence of publication bias was detected through funnel plot analyses.
\end{abstract}

Conclusions: SR treatment of stage I NSCLC patients was associated with a lower LR rate and longer survival as compared to ablation.

Keywords: Sublobar resection, Ablation, Lung cancer, Meta-analysis

\section{Background}

Non-small-cell lung cancer (NSCLC) is the deadliest form of cancer globally [1-3], and many patients with early-stage NSCLC can undergo curative surgical resection following tumor detection via chest computed tomography (CT) screening [4-6]. Lobectomy with systematic mediastinal lymphadenectomy is the standard approach to treating stage I NSCLC [7-9]. However, in many cases, patients are elderly or have a history of comorbidities such as pulmonary dysfunction or

*Correspondence: yayonghuang@yeah.net

2 Department of Radiology, Xuzhou Central Hospital, 199 South Jiefang Road, Xuzhou, China

Full list of author information is available at the end of the article atherosclerosis that may preclude their ability to undergo invasive surgical treatment $s$ [4-6].

While sublobar resection (SR) is generally less effective as a curative procedure relative to lobectomy [4], it can better preserve patient pulmonary function [7-9]. In stage I NSCLC patients with tumors $\leq 2 \mathrm{~cm}$ in size, SR yields similar survival outcomes to lobectomy $[5,6]$. However, $20-30 \%$ of stage I NSCLC patients are unable to undergo SR owing to their poor performance status [10-17]. In these patients, percutaneous ablation represents the most minimally invasive treatment strategy [10-17]. While prior studies have compared the relative efficacy of SR and ablation in stage I NSCLC patients, the majority of these analyses have been retrospective in design [10-17]. Conducting a meta-analysis would thus original author(s) and the source, provide a link to the Creative Commons licence, and indicate if changes were made. The images or other third party material in this article are included in the article's Creative Commons licence, unless indicated otherwise in a credit line to the material. If material is not included in the article's Creative Commons licence and your intended use is not permitted by statutory regulation or exceeds the permitted use, you will need to obtain permission directly from the copyright holder. To view a copy of this licence, visit http://creativecommons.org/licenses/by/4.0/. The Creative Commons Public Domain Dedication waiver (http://creativecommons.org/publicdomain/zero/1.0/) applies to the data made available in this article, unless otherwise stated in a credit line to the data. 
represent an effective means of reducing potential bias and increasing statistical power associated in order to develop more reliable conclusions.

The present meta-analysis was therefore designed to compare the relative safety and efficacy of SR and ablation for the treatment of individuals with stage I NSCLC.

\section{Methods}

\section{Study selection}

This meta-analysis was conducted in accordance with the Preferred Reporting Items for Systematic reviews and Meta-Analyses (PRISMA) statement. This work was registered in https://inplasy.com/ (No. INPLASY202110075).

Relevant studies published as of November 2020 in the Cochrane Library, Embase, and PubMed databases were identified via the following search strategy: $\quad(((($ radiofrequency[Title/Abstract] $) \quad$ OR (microwave[Title/Abstract])) OR (cryoablation[Title/ Abstract])) OR (ablation[Title/Abstract])) AND ((() surgery[Title/Abstract]) OR (resection[Title/ Abstract])) OR (Video assisted thoracoscopic surgery[Title/Abstract])) OR (VATS[Title/Abstract]))) AND ((lung cancer[Title/Abstract]) OR (NSCLC[Title/ Abstract])).

Studies eligible for inclusion in this meta-analysis included (a) randomized controlled trials (RCTs) or retrospective analyses, (b) studies of stage I NSCLC patients, (c) studies in which SR and ablation were compared to treat NSCLC. Studies published in any language were eligible for inclusion.

Studies were excluded if they were (a) non-comparative studies, (b) animal or preclinical studies, or (c) reviews.

\section{Data extraction}

Baseline patient data, baseline study data, and treatmentrelated data were independently extracted by two investigators. Discussion with a third author was used to resolve any inconsistencies.

\section{Quality assessment}

Study quality was assessed independently by two researchers. RCT quality was assessed with the Cochrane risk of bias tool based upon selection, performance, detection, attrition, reporting, and other biases. All other studies were evaluated using the 9-point NewcastleOttawa scale (NOS) [18], with high-quality studies being those scoring $\geq 6$ points.

\section{Endpoints and definitions}

Meta-analysis endpoints included rates of complications, recurrence rates, duration of postoperative hospitalization, and patient survival, with survival as the primary study endpoint.

For this study, SR was defined as both segmental and wedge resection operations [10-17]. Both local recurrence (LR) and distant recurrence (DR) outcomes were evaluated, with the latter of these including intra- and extra-pulmonary DR [11, 12]. Analyzed survival outcomes included overall, progression-free, and cancerspecific survival (OS, PFS, and CSS, respectively).

\section{Meta-analysis}

RevMan v5.3 was used for all meta-analyses. Pooled odds ratios (ORs) and corresponding 95\% confidence intervals (CIs) were calculated via the Mantel-Haenszel method for dichotomous variables, whereas continuous variables were analyzed based upon mean difference (MD) values and 95\% CIs. Pooled survival duration was assessed based upon hazard ratios (HRs) and 95\% CIs. Study heterogeneity was evaluated based upon $\mathrm{X}^{2}$ tests and the $\mathrm{I}^{2}$ statistic, with $\mathrm{I}^{2}>50 \%$ being indicative of significant heterogeneity. When significant heterogeneity was detected, data were analyzed with a random-effects model, whereas fixed-effects models were otherwise utilized. Sources of heterogeneity were assessed with sensitivity and subgroup analyses, and funnel plots were used to test the risk of publication bias.

\section{Results \\ Study characteristics}

The initial search strategy identified 816 potentially relevant articles, of which eight were ultimately included in our final meta-analysis (Fig. 1). These were all retrospective studies with NOS scores between 6 and 8 points (Table 1), incorporating 679 patients treated by SR and 468 that underwent ablation (Tables 1,2). In five studies, patients underwent radiofrequency ablation (RFA) $[10,11,13,15,16]$, while in three studies, wedge resection and ablation treatments were compared [11, 12, 17]. Treatment-associated data for these studies are shown in Table 2.

\section{Recurrence}

LR was reported in three studies $[11,12,16]$, with pooled LR rates being significantly lower in the SR group $(5.0 \%$ vs. $25.4 \%, P<0.0001$, Fig. 2 a). No significant heterogeneity pertaining to this endpoint was detected $\left(\mathrm{I}^{2}=0 \%\right)$.

DR was reported in two studies [11, 12], and pooled DR rates were similar between groups $(25.7 \%$ vs. $23.1 \%$, $P=0.75$, Fig. $2 \mathrm{~b})$. No significant heterogeneity pertaining to this endpoint was detected $\left(\mathrm{I}^{2}=0 \%\right)$. 


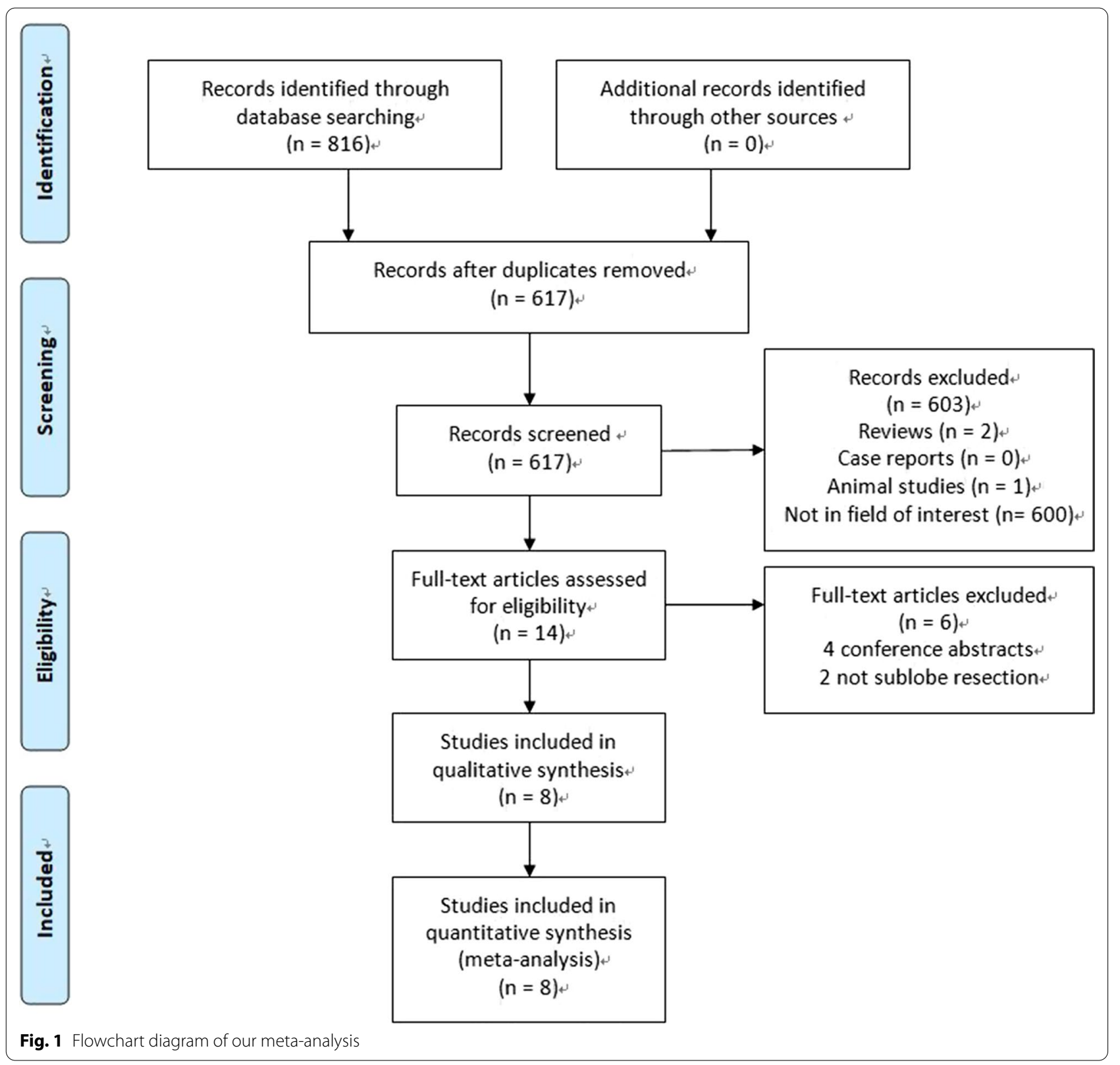

\section{Survival}

OS was reported in all studies, and the pooled OS HR was more favorable in the SR group (HR: 1.23 ; 95\% CI: $1.13-1.33, P<0.00001$, Fig. 2c). This endpoint was associated with significant heterogeneity $\left(\mathrm{I}^{2}=73 \%\right)$, but omitting individual studies in a sensitivity analysis had no significant impact on overall heterogeneity.

PFS was reported in six studies [10-13, 15, 16], with the pooled PFS HR being more favorable in the SR group (HR: 1.34; 95\% CI: $1.15-1.55, P=0.0002$,
Fig. 2d). This endpoint was associated with significant heterogeneity $\left(\mathrm{I}^{2}=76 \%\right)$, but omitting individual studies in a sensitivity analysis had no significant impact on overall heterogeneity.

CSS was reported in five studies [10, 11, 14, 16, 17], with the pooled CSS HR being more favorable in the SR group (HR: 1.39; 95\% CI: 1.15-1.70, $P=0.0009$, Fig. 2e). This endpoint was associated with significant heterogeneity $\left(\mathrm{I}^{2}=74 \%\right)$, but omitting individual studies in a sensitivity analysis had no significant impact on overall heterogeneity. 
Table 1 Characteristics of the included studies

\begin{tabular}{|c|c|c|c|c|c|c|c|c|}
\hline Study/year/country & Groups & Sample size & Male/female & Age (years) & $\begin{array}{l}\text { Tumor size } \\
(\mathrm{mm})\end{array}$ & $\begin{array}{l}\text { FEV1/ } \\
\text { predicted } \\
(\%)\end{array}$ & CCMI & NOS \\
\hline \multirow[t]{2}{*}{ Alexander/2013/USA [10] } & SR & 28 & $12 / 16$ & 74 & NG & 54 & 42.61 & 7 \\
\hline & Ablation & 56 & $24 / 32$ & 78 & NG & 52 & 42.45 & \\
\hline \multirow[t]{2}{*}{ Ambrogi/2015/Italy [11] } & SR & 59 & $46 / 13$ & 70 & 26 & 47 & NG & 8 \\
\hline & Ablation & 62 & $45 / 17$ & 76 & 23 & 49 & NG & \\
\hline \multirow[t]{2}{*}{$\mathrm{Hu/2020/China} \mathrm{[12]}$} & $\mathrm{SR}$ & 155 & $103 / 52$ & 78 & 25 & 71 & $4^{\mathrm{a}}$ & 8 \\
\hline & Ablation & 68 & $44 / 24$ & 83 & 23 & 63 & $4^{\mathrm{a}}$ & \\
\hline \multirow[t]{2}{*}{ Iguchi/2020/Japan [13] } & SR & 193 & $101 / 92$ & 67 & 16 & NG & $2.78^{\mathrm{a}}$ & 7 \\
\hline & Ablation & 38 & $22 / 16$ & 75 & 22 & NG & $3.03^{\mathrm{a}}$ & \\
\hline \multirow[t]{2}{*}{ Kwan/2014/USA [14] } & SR & 69 & $36 / 33$ & NG & NG & NG & NG & 6 \\
\hline & Ablation & 99 & $26 / 73$ & NG & NG & NG & NG & \\
\hline \multirow[t]{2}{*}{ Safi/2015/Germany [15] } & SR & 42 & $27 / 15$ & 70 & 19 & 69 & 6.2 & 8 \\
\hline & Ablation & 25 & $18 / 7$ & 71 & 22 & 67 & 5.5 & \\
\hline \multirow[t]{2}{*}{ Zemlyak/2010/USA [16] } & SR & 25 & $9 / 16$ & 66 & NG & 65 & NG & 7 \\
\hline & Ablation & 12 & $7 / 5$ & 74 & NG & 64 & $N G$ & \\
\hline \multirow[t]{2}{*}{ Zeng/2020/China [17] } & SR & 108 & $58 / 60$ & 70.2 & NG & NG & NG & 7 \\
\hline & Ablation & 108 & $44 / 64$ & 68.1 & NG & NG & NG & \\
\hline
\end{tabular}

CCMI Charlson Comorbidity Index, SR sublobar resection, NG not given, NOS Newcastle-Ottawa scale

${ }^{a}$ The Charlson comorbidity index was significant lower in SR group

Table 2 Characteristics of the treatment outcomes

\begin{tabular}{|c|c|c|c|c|c|c|c|}
\hline Study & Groups & SR methods & Ablation & LR (\%) & DR (\%) & Complication (\%) & Hospital stay \\
\hline \multirow[t]{2}{*}{ Alexande [10] } & SR & Segmental and wedge & RFA & Not given & Not given & $53.6 \%$ & Not given \\
\hline & Ablation & & & Not given & Not given & $62.3 \%$ & Not given \\
\hline \multirow[t]{2}{*}{ Ambrogi [11] } & SR & Wedge & RFA & $1.7 \%$ & $11.9 \%$ & $27.1 \%$ & 6 days \\
\hline & Ablation & & & $22.6 \%$ & $11.3 \%$ & $21.0 \%$ & 2 days \\
\hline \multirow[t]{2}{*}{$\mathrm{Hu}[12]$} & SR & Wedge & Microwave & $5.2 \%$ & $31.0 \%$ & Not given & Not given \\
\hline & Ablation & & & $26.5 \%$ & $33.8 \%$ & Not given & Not given \\
\hline \multirow[t]{2}{*}{ Iguchi [13] } & $\mathrm{SR}$ & Segmental and wedge & RFA & Not given & Not given & 368 days & 16 days \\
\hline & Ablation & & & Not given & Not given & 222 days & 6.5 days \\
\hline \multirow[t]{2}{*}{ Kwan [14] } & $\mathrm{SR}$ & Segmental and wedge & No details & Not given & Not given & 232 days & Not given \\
\hline & Ablation & & & Not given & Not given & 110 days & Not given \\
\hline \multirow[t]{2}{*}{ Safi [15] } & $\mathrm{SR}$ & Segmental and wedge & RFA & Not given & Not given & 9.8 months & Not given \\
\hline & Ablation & & & Not given & Not given & 5.6 months & Not given \\
\hline \multirow[t]{2}{*}{ Zemlyak [16] } & SR & Segmental and wedge & RFA & $12 \%$ & Not given & 0 & Not given \\
\hline & Ablation & & & $33.3 \%$ & Not given & $66.7 \%$ & Not given \\
\hline \multirow[t]{2}{*}{ Zeng [17] } & SR & Wedge & No details & Not given & Not given & 194 days & Not given \\
\hline & Ablation & & & Not given & Not given & 86 days & Not given \\
\hline
\end{tabular}

$L R$ local recurrence, $D R$ distant recurrence, $R F A$ radiofrequency ablation, $S R$ sublobar resection

\section{Complications}

Complication rates were reported in three studies [10, $11,16]$, and were similar in both groups $(27.7 \%$ vs. $43.8 \%$, $P=0.27$, Fig. 2f). This endpoint was associated with significant heterogeneity $\left(\mathrm{I}^{2}=91 \%\right)$, and the omission of the study conducted by Zemlyak et al.[16] eliminated this heterogeneity. When this study was omitted, pooled complication rates remained similar between groups $(P=0.98)$.

\section{Duration of postoperative hospitalization}

Two studies reported the duration of postoperative hospitalization for treated patients $[11,13]$. The pooled duration of postoperative hospitalization was significantly 


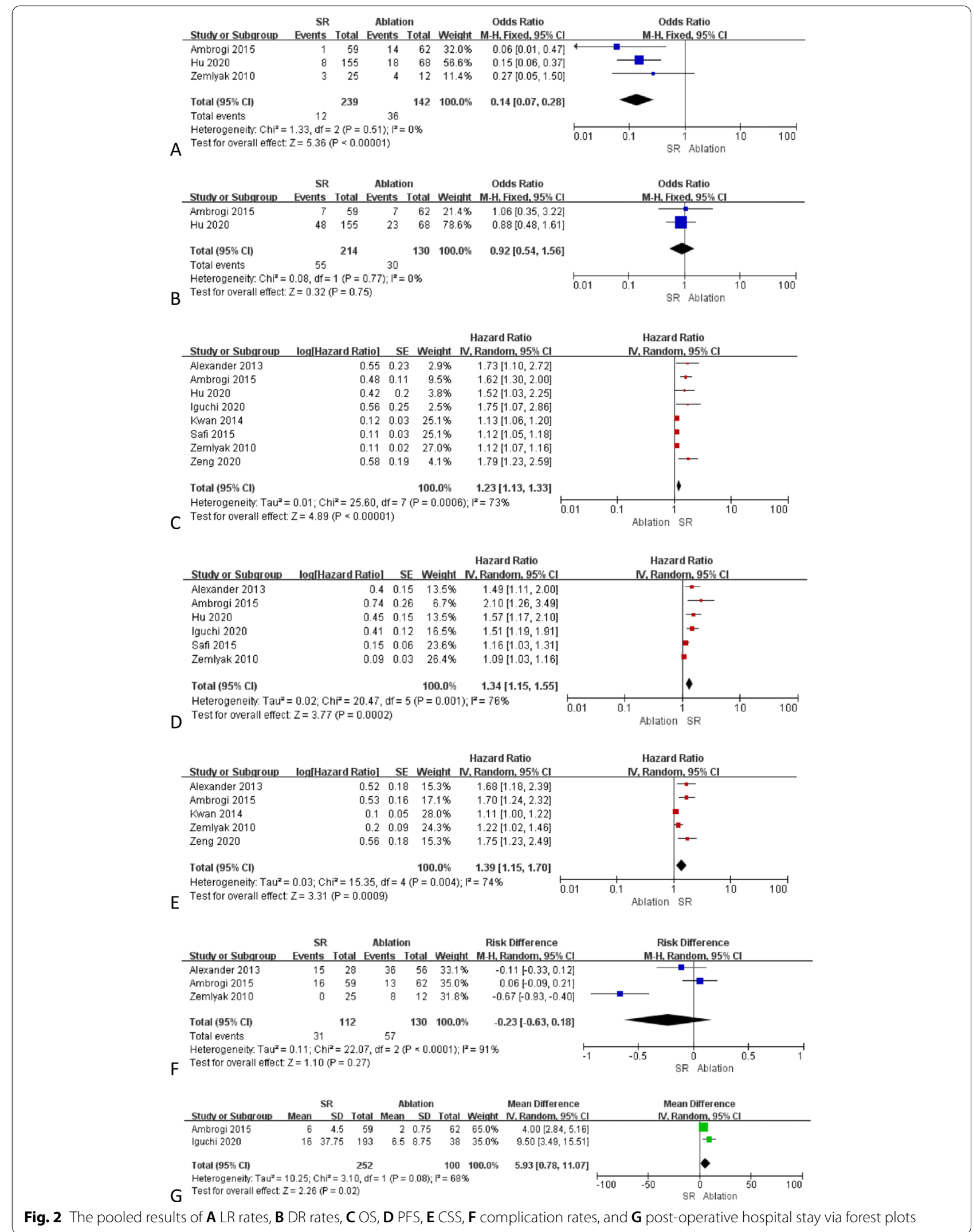


lower in the ablation group relative to the SR group (MD: 5.93; 95\% CI: $0.78-11.07, P=0.02$, Fig. 2h). This endpoint was associated with significant heterogeneity $\left(\mathrm{I}^{2}=68 \%\right)$.

\section{Subgroup analyses}

Five articles specifically compared SR and RFA as approaches to treating stage I NSCLC $[10,11,13,15,16]$, and in these studies, pooled HRs pertaining to OS, PFS, and CSS were all favorable in the SR group (Table 3).

Three articles specifically compared wedge resection and ablation as approaches to treating stage I NSCLC $[11,12,17]$, and in these studies, pooled HRs pertaining to OS, PFS, and CSS were all favorable in the SR group (Table 4).

\section{Publication bias}

No evidence of publication bias was detected through funnel plot analyses.

\section{Discussion}

Current American College of Chest Physicians clinical practice guidelines suggest that stage I NSCLC patients undergo lobectomy with systematic mediastinal lymph node dissection when possible. SR is the preferred treatment in patients considered at high risk for lobar resection, although some studies have suggested that lobectomy and SR are associated with similar outcomes in those with stage I NSCLC $[19,20]$. When patients are unable to tolerate SR, it is typically recommended that patients undergo ablation [14].

The present meta-analysis compared the relative safety and efficacy of SR and ablation for the treatment of stage I NSCLC. When comparing recurrence rates between these two patient groups, our analysis revealed LR rates to be significantly lower in the SR group (5.0\% vs. $25.4 \%$, $P<0.0001)$. This suggests that ablation cannot fully eliminate tumors, consistent with the fact that this approach is less effective when treating tumors adjacent to large vessels $>3 \mathrm{~mm}$ in diameter or to a bronchus $>2 \mathrm{~mm}$ in diameter owing to heat-sink effects [13]. However, there are certain advantages to the ablation procedure, including the fact that it can be performed repeatedly and can be employed to treat both synchronous and metachronous lesions [21]. We observed similar pooled DR rates in both patient groups $(25.7 \%$ vs. $23.1 \%, P=0.75)$, indicating that both SR and ablation are limited in their ability to control systematic tumor growth. Postoperative chemotherapy may represent a viable means of lowering DR rates [22].

Stage I NSCLC patient treatment primarily focuses on improving patient survival rates. We therefore analyzed OS, PFS, and CSS outcomes, revealing substantial variability among studies. Kwan et al. [14] detected similar OS $(P=0.695)$ and CSS $(P=0.819)$ between the SR and ablation groups following a propensity score-matched analysis, while Safi et al. [15] similarly found OS $(P=0.28)$ and PFS $(P=0.09)$ to be comparable in these two treatment groups, and Zemlyak et al. [16] found these two groups to exhibit similar OS $(P>0.05)$, CSS $(P>0.05)$, and PFS $(P>0.05)$ outcomes. While 2-year OS rates in the studies conducted by Kwan et al. and Safi et al. ranged from $66-85 \%$ and $62-74 \%$ in SR and ablation groups, respectively $[14,15]$, in other studies the survival of patients in the SR group was significantly longer than that of patients in the ablation group $[11-13,17]$. These differences may be attributable to differences in sample size, tumor size, or patient selection criteria among studies. In pooled OS, PFS, and CSS analyses, survival rates were better in the SR group, and subgroup analyses further confirmed that

Table 3 Meta-analytic pooled results of survival based on the RFA versus SR

\begin{tabular}{lllll}
\hline & Number of studies & HR (95\% Cl) & Heterogeneity & Favor \\
\hline Overall survival & 5 & $1.24(1.11,1.38), P=0.0002$ & $P^{2}=77 \%$ & $S R$ \\
Progression-free survival & 5 & $1.30(1.11,1.51), P=0.001$ & $P^{2}=75 \%$ & $S R$ \\
Cancer-specific survival & 4 & $1.33(1.09,1.62), P=0.005$ & $P^{2}=73 \%$ & $S R$ \\
\hline
\end{tabular}

$H R$ hazard ratio, $\mathrm{Cl}$ confidential interval, $R F A$ radiofrequency ablation, $S R$ sublobar resection

Table 4 Meta-analytic pooled results of survival based on the ablation versus wedge resection

\begin{tabular}{lllll}
\hline & Number of studies & HR $(\mathbf{9 5} \% \mathbf{C l})$ & Heterogeneity & Favor \\
\hline Overall survival & 3 & $1.63(1.38,1.93), P<0.00001$ & $P^{2}=0 \%$ & $S R$ \\
Progression-free survival & 2 & $1.69(1.31,2.18), P<0.00001$ & $P^{2}=0 \%$ & $S R$ \\
Cancer-specific survival & 2 & $1.72(1.36,2.18), P<0.00001$ & $P^{2}=0 \%$ & $S R$
\end{tabular}

$H R$ hazard ratio, $\mathrm{Cl}$ confidential interval, $S R$ sublobar resection 
wedge resection was associated with superior OS, PFS, and CSS outcomes relative to ablation.

The significantly prolonged survival observed following SR was primarily attributable to the significantly lower LR rate in these patients. In addition, an analysis of 100 NSCLC patients with tumors $<1 \mathrm{~cm}$ in diameter indicated that $5 \%$ of these patients exhibited lymph node involvement, suggesting that such involvement should still be considered even in those with mall lesions [21]. SR thus offers the additional advantage of facilitating lymph node sampling at time of surgery, enabling clinicians to more precisely stage patients and to thereby guide treatment [21].

Pooled complication rates were similar in both groups, suggesting that both SR and ablation exhibit similar safety profiles when used to treat those with stage I NSCLC. However, patients who underwent ablation experienced significantly shorter postoperative hospitalization relative to patients treated via SR, owing to the fact that ablation is a less invasive procedure not requiring the use of general anesthesia.

There were multiple limitations to this analysis. For one, the articles included in this meta-analysis were retrospective in nature, rendering them susceptible to selection bias. Additional RCTs will therefore be required to validate and expand upon these data. Secondly, certain study endpoints were associated with significant heterogeneity, and while the sources of such heterogeneity were identified when possible, additional RCTs will be essential to establish definitive research results. Third, preoperative imaging analyses were used for the evaluation of mediastinal and hilar lymph nodes in the context of RFA without any pathologic verification, potentially leading to an underestimation of accurate patient staging, thus biasing survival outcome data.

\section{Conclusion}

In summary, SR was associated with lower LR rats and prolonged survival relative to ablation when used to treat stage I NSCLC patients.

\section{Abbreviations \\ CT: Computed tomography; CSS: Cancer-specific survival; DR: Distant recurrence; LR: Local recurrence; NSCLC: Non-small-cell lung cancer; PFS: Progression-free survival; OS: Overall survival; SR: Sublobar resection; WR: Wedge resection.}

\section{Acknowledgements}

None.

\section{Authors' contributions}

YYH designed this work. YL and FY searched the articles. YL, FY, and TW performed the data extraction and statistical analyses. YL wrote this article. All authors read and approved the final manuscript.
Funding

None.

Availability of data and materials

The data that support the findings of this study are available from the corresponding author upon reasonable request.

\section{Declarations}

Ethics approval and consent to participate

This is a meta-analysis and ethics approval and consent to participate are not required.

\section{Consent for publication}

This is a meta-analysis and consent for publication is not required.

\section{Competing interests}

The authors declare that they have no competing interests.

\section{Author details}

${ }^{1}$ Sichuan Key Laboratory of Medical Imaging and Department of Radiology, Affiliated Hospital of North Sichuan Medical College, Nanchong, China. ${ }^{2}$ Department of Radiology, Xuzhou Central Hospital, 199 South Jiefang Road, Xuzhou, China.

Received: 18 February 2021 Accepted: 3 February 2022

Published online: 11 February 2022

\section{References}

1. Herbst RS, Morgensztern D, Boshoff C. The biology and management of non-small cell lung cancer. Nature. 2018;553:446-54.

2. Rafei H, El-Bahesh E, Finianos A, et al. Immune-based therapies for nonsmall cell lung cancer. Anticancer Res. 2017;37:377-87.

3. Akhurst T. Staging of non-small-cell lung cancer. PET Clin. 2018;13:1-10.

4. Zhang $Y$, Sun $Y$, Wang R, et al. Meta-analysis of lobectomy, segmentectomy, and wedge resection for stage I non-small cell lung cancer. J Surg Oncol. 2015;111:334-40.

5. Fan J, Wang L, Jiang GN, et al. Sublobectomy versus lobectomy for stage I non-small-cell lung cancer, a meta-analysis of published studies. Ann Surg Oncol. 2012;19:661-8.

6. Namkoong M, Moon Y, Park JK. Lobectomy versus sublobar resection in non-lepidic small-sized non-small cell lung cancer. Korean J Thorac Cardiovasc Surg. 2017;50:415-23.

7. Fu YF, Zhang M, Wu WB, et al. Coil localization-guided video-assisted thoracoscopic surgery for lung nodules. J Laparoendosc Adv Surg Tech A. 2018;28:292-7.

8. Teng F, Wang ZS, Wu AL, et al. Computed tomography-guided coil localization for video-assisted thoracoscopic surgery of sub-solid lung nodules: a retrospective study. ANZ J Surg. 2019;89:E514-8.

9. Yang ZJ, Liang YH, Li M, et al. Preoperative computed tomographyguided coil localization of lung nodules. Minim Invasive Ther Allied Technol. 2020;29:28-34.

10. Alexander ES, Machan JT, Ng T, Breen LD, DiPetrillo TA, Dupuy DE. Cost and effectiveness of radiofrequency ablation versus limited surgical resection for stage I non-small-cell lung cancer in elderly patients: is less more? J Vasc Interv Radiol. 2013:24:476-82.

11. Ambrogi MC, Fanucchi O, Dini P, et al. Wedge resection and radiofrequency ablation for stage I nonsmall cell lung cancer. Eur Respir J. 2015;45:1089-97.

12. $\mathrm{Hu} \mathrm{H}$, Zhai B, Liu R, et al. Microwave ablation versus wedge resection for stage I non-small cell lung cancer adjacent to the pericardium: propensity score analyses of long-term outcomes. Cardiovasc Intervent Radiol. 2020. https://doi.org/10.1007/s00270-020-02601-7.

13. Iguchi T, Hiraki T, Matsui Y, et al. Survival outcomes of treatment with radiofrequency ablation, stereotactic body radiotherapy, or sublobar resection for patients with clinical stage I non-small-cell lung cancer: a single-center evaluation. J Vasc Interv Radiol. 2020;31:1044-51. 
14. Kwan SW, Mortell KE, Talenfeld AD, et al. Thermal ablation matches sublobar resection outcomes in older patients with early-stage non-small cell lung cancer. J Vasc Interv Radiol. 2014;25:1-9.e1.

15. Safi S, Rauch G, Op den Winkel J, et al. Sublobar resection, radiofrequency ablation or radiotherapy in stage I non-small cell lung cancer. Respiration. 2015;89:550-7.

16. Zemlyak A, Moore WH, Bilfinger TV. Comparison of survival after sublobar resections and ablative therapies for stage I non-small cell lung cancer. J Am Coll Surg. 2010;211:68-72.

17. Zeng C, Lu J, Tian Y, et al. Thermal ablation versus wedge resection for stage I non-small cell lung cancer based on the eighth edition of the TNM classification: a population study of the us seer database. Front Oncol. 2020;10:571684.

18. Wells GA, Shea BJ, O'Connell D, et al. The Newcastle-Ottawa scale (NOS) for assessing the quality of non-randomized studies in meta-analysis. Appl Eng Agric. 2000;18:727-34.

19. Okada M, Koike T, Higashiyama M, et al. Radical sublobar resection for small-sized non-small cell lung cancer: a multicenter study. J Thorac Cardiovasc Surg. 2006;132:769-75.

20. Koike T, Yamato Y, Yoshiya K, et al. Intentional limited pulmonary resection for peripheral T1NOMO small-sized lung cancer. J Thorac Cardiovasc Surg. 2003;125:924-8.

21. Chen $\mathrm{S}$, Yang $\mathrm{S}$, Xu S, et al. Comparison between radiofrequency ablation and sublobar resections for the therapy of stage I non-small cell lung cancer: a meta-analysis. PeerJ. 2020;8:e9228.

22. Pei J, Han B, Zhang J, et al. Multivariate survival analysis of patients with stage I NSCLC. Zhongguo Fei Ai Za Zhi. 2011;14:926-32.

\section{Publisher's Note}

Springer Nature remains neutral with regard to jurisdictional claims in published maps and institutional affiliations.

Ready to submit your research? Choose BMC and benefit from:

- fast, convenient online submission

- thorough peer review by experienced researchers in your field

- rapid publication on acceptance

- support for research data, including large and complex data types

- gold Open Access which fosters wider collaboration and increased citations

- maximum visibility for your research: over $100 \mathrm{M}$ website views per year

At BMC, research is always in progress.

Learn more biomedcentral.com/submissions 